\title{
Local MTM-SVD based Spectrum Sensing in SIMO OFDM Cognitive Radio under Bandwidth Constraint
}

\author{
Owayed A. Alghamdi and Mosa A. Abu-Rgheff \\ Mobile Communications Research Group \\ University of Plymouth \\ Plymouth PL4 8AA, UK \\ owayed.alghamdi@plymouth.ac.uk
}

\begin{abstract}
In this paper, we propose using local multitapersingular value decomposition (Local-MTM-SVD) for spectrum sensing in OFDM-based cognitive radio (CR) systems. The spectrum sensing locality in this technique is enhanced by using single input at the primary (PR) transmitter and multi output antenna system (SIMO) at the different CR nodes (sensors). The global decision is declared using the OR rule at the $C R$ basestation based on hard cooperation. This technique reduces the bandwidth of the feedback channel, and for powerful spectrum sensing technique; the MTM-SVD is used. Our proposed technique is examined using software simulation and theoretical analysis. The results show that there is a significant improvement in the probability of detection compared to using MTM only, or using the periodogram with single antenna in both AWGN and Rayleigh flat fading environments. We found the number of singular value squares that are useful in the technique is equal to or smaller than 2 when the total number of generated singular values is 4 , and the MTM only technique tends to offer same performance as the Local-MTM-SVD technique using single antenna in the same conditions. The choice of the decision threshold level depends on the number of singular value squares which we used in the decision statistic.
\end{abstract}

Keywords - cognitive radio; spectrum sensing; multitaper spectrum estimation method; singular value decomposition; cooperative spectrum sensing.

\section{INTRODUCTION}

Multitaper singular-value decomposition (MTM-SVD)[13], is a promising technique in CR spectrum sensing [4]. However practical implementation of this method requires each CR node (sensor) to send its information about the RF environment from different tapers at each frequency bin at each sensing time to the main CR basestation. Such requirements need to use huge feedbacks overhead [5]. Thus MTM-SVD can be classified as soft cooperation [6], but with multi measurements. Furthermore the need for SVD at the CR base station increases the complexity when the number of $\mathrm{CR}$ nodes is large.

In our previous paper [7], we combined Haykin theoretical work on spectrum sensing using multitaper method with singular value decomposition detection of PR's transmission in Gaussian noise environment. The paper presents results concerning the behavior of MTM scheme to problems such as bias, variance and out of band leakage that are associated with conventional spectrum sensing, and the SVD's ability for denoising. But no attempt was made to research minimizing the numbers of information to be sent to the CR basestation. Our work in [8], shows that the half time bandwidth $N W=4$, and number of tapers $K=5$ are the MTM parameters that give the highest probability of detection in 64-IFFT/FFT- based CR systems.

In wireless communications, the main objective of using multi antenna at the receiver is to resolve the deep fading problems. Choosing suitable distances between the different antennas insures that, the fading events are independent through the different antennas. We then use one of the classical combining techniques to combine the different outputs from the different antennas [9].

Generally using multi antennas in spectrum sensing at each $\mathrm{CR}$ node (sensor) produces space diversity. The wireless channel in such kind of space diversity is called Single Input Multi Output (SIMO) channel.

In this paper, we propose using the local-MTM-SVD with single input multi output antenna system (SIMO) at each CR node (sensor), and sending the CR nodes(sensors) decisions to the $\mathrm{CR}$ basestation using binary digit $\{0,1\}$, or hard cooperation [9]. Finally the final decision will be declared to the CR nodes using the OR cooperation rule [10], which will be performed at the CR basestation. Of course minimizing the overhead feedbacks using narrow control channel allows usage of an efficient spectrum sensing technique MTM-SVD without the need of sending a large number of measurements through the CR network. Furthermore there is no more need for the SVD processing at the CR basestation thus decreasing the complexity at the CR basestation side.

The rest of the paper is organized as follows: Section II defines the model for the system under consideration. Section III considers the theoretical background of the MTM, periodogram, the local-MTM-SVD, and OR rule cooperation. 
Section IV presents the results and Section V concludes the paper.

\section{SYSTEM MODEL}

Our system model consists of a single PR transmit/receive node, transmitting QPSK-OFDM signal in the sub-band between $f_{a}$ and $f_{b}$, and a number of OFDM-based CR nodes (sensors) that detect the PR user's signal and relay information about the detected signal to CR basestation that applies certain cooperation algorithm to decide whether the PR' signal is present or absent in the searched frequency band as shown in Fig. 1.

\section{LOCAL-MTM-SVD SPECTRUM SENSING}

The received $\mathrm{PR}$ signal at a $\mathrm{CR}$ node (sensor) is sampled to generate a finite discrete time samples serieses $\left\{x_{t}^{m} ; t=\right.$ $0,1, \ldots, N-1, m=0,1, \ldots, M-1\}$, where $m$ denotes the antenna number, and $t$ is time index. The discrete time samples are dot multiplied with different tapers (tapers are Discrete Prolate Slepian Sequences). The product is applied to a Fourier Transform to compute the energy concentrated in the bandwidth $(-W, W)$ centered at frequency $f$.

In order to evaluate the performance of the local-MTMSVD technique, we review two different types of the probabilities at each frequency bin $f_{i}$, the probability of detection $P_{D}\left(f_{i}\right)$ and the probability of false alarm $P_{F}\left(f_{i}\right)$. $P_{D}\left(f_{i}\right)$, is the probability that the CR node (sensor) decides correctly the presence of the PR's signal, and $P_{F}\left(f_{i}\right)$ is the probability that the CR node (sensor) decides the PR's signal is present when it is absent.

The binary hypothesis test for CR spectrum sensing at the $l^{\text {th }}$ time, and the $m^{\text {th }}$ antenna branch is given by:

$$
\begin{aligned}
& \mathcal{H}_{0}: \quad x^{m}(l)=w^{m}(l) \\
& \mathcal{H}_{1}: \quad x^{m}(l)=h^{m} s(l)+w^{m}(l)
\end{aligned}
$$

where $l=0,1, \ldots, L-1$ is OFDM block's index, $x^{m}(l), w^{m}(l)$ and $s(l)$ denote the CR received, noise at the branch $m$, and PR transmitted samples. The transmitted PR signal is distorted by the zero mean additive white Gaussian noise $w^{m}(l) \sim \mathcal{C N}\left(0, \sigma_{\text {noise }-m}^{2}\right)$ at the output from the different antenna branches. Additionally the channel between PR transmitter and the $m^{t h}$ antenna of the CR receiver is subjected to Rayleigh flat fading. The channel gain $h^{m}$ is assumed to be constant during the sensing time and independent for each antenna. Note that at each frequency bin of CR FFT $\mathcal{H}_{0}$ indicates no PR signal present while $\mathcal{H}_{1}$ means there is a PR signal present.

The time instant $l$ comes from the samples over different OFDM blocks; and time instant $t$ comes from the samples from the same OFDM block (i.e., IFFT/FFT samples).

In MTM; for $K$ orthonormal tapers, there will be $K$ different eigenspectrums produced from the $m^{\text {th }}$ antenna during the $l^{t h}$ OFDM block defined as:

$$
Y_{k}^{m}\left(f_{i}\right)=\sum_{t=0}^{N-1} v_{t}^{(k)}(N, W)\left(h^{m} s_{t}(l)+w_{t}^{m}(l)\right) e^{-j 2 \pi f_{i} t}
$$

where $v_{t}^{(k)}(N, W)$ is the $k^{t h}$ discrete prolate Slepian sequence (DPSS), and $\lambda_{k}(N, W)$ is the associated eigenvalue. $f_{i}=$ $0, \frac{1}{N}, \frac{2}{N}, \ldots, \frac{N-1}{N}$ are the normalized frequency bins.

Then the spectrum estimates using MTM with single antenna is defined as :

$$
S_{M T M}^{m}\left(f_{i}\right)=\frac{\sum_{k=0}^{K-1} \lambda_{k}(N, W)\left|Y_{k}^{m}\left(f_{i}\right)\right|^{2}}{\sum_{k=0}^{K-1} \lambda_{k}(N, W)}
$$

On the other hand, the periodogram method using single antenna, when the samples are taken at uniform time spacing, gives the power spectrum density estimation as :

$$
S_{P E}^{m}\left(f_{i}\right)=\frac{1}{N}\left|\sum_{t=0}^{N-1}\left(h^{m} s_{t}(l)+w_{t}^{m}(l)\right) e^{-j 2 \pi f_{i} t}\right|^{2}
$$

Now when a number of $M$ antennas are used, the complex eigenspectrums row vector $\boldsymbol{r}^{m}\left(f_{i}\right)$, which is resulted from the $m^{\text {th }}$ antenna using the different tapers, is defined as:

$$
\boldsymbol{r}^{m}\left(f_{i}\right)=\left[Y_{0}^{m}\left(f_{i}\right) Y_{1}^{m}\left(f_{i}\right) Y_{2}^{m}\left(f_{i}\right) \ldots . Y_{K-1}^{m}\left(f_{i}\right)\right]
$$

Where $Y_{k}^{m}(f)$ denotes the eigenspectrum which is produced from the $m^{\text {th }}$ antenna using the $k^{\text {th }}$ taper. The complex eigenspectrums column vector $\boldsymbol{c}^{k}(f)$, which is resulted from the $k^{\text {th }}$ taper using different antennas, is defined as:

$$
\boldsymbol{c}^{k}\left(f_{i}\right)=\left[Y_{k}^{0}\left(f_{i}\right) Y_{k}^{1}\left(f_{i}\right) Y_{k}^{2}\left(f_{i}\right) \ldots . Y_{k}^{M-1}\left(f_{i}\right)\right]^{T}
$$

where $T$, is the vector transpose. Now, the spatio-temporal complex matrix $\boldsymbol{A}\left(f_{i}\right)$ can be constructed using either complex eigenspectrums row vectors $\boldsymbol{r}^{m}\left(f_{i}\right)$, or column vectors $\boldsymbol{c}^{k}\left(f_{i}\right)$ as follow:

$$
\boldsymbol{A}\left(f_{i}\right)=\left[\begin{array}{c}
\boldsymbol{r}^{\mathbf{0}}\left(f_{i}\right) \\
\boldsymbol{r}^{\mathbf{1}}\left(f_{i}\right) \\
\boldsymbol{r}^{\mathbf{2}}\left(f_{i}\right) \\
\cdot \\
\cdot \\
\cdot \\
\boldsymbol{r}^{\boldsymbol{M - 1}}\left(f_{i}\right)
\end{array}\right]
$$

$$
\boldsymbol{A}\left(f_{i}\right)=\left[\boldsymbol{c}^{\mathbf{0}}\left(f_{i}\right) \boldsymbol{c}^{\mathbf{1}}\left(f_{i}\right) \boldsymbol{c}^{\mathbf{2}}\left(f_{i}\right) \ldots . \boldsymbol{c}^{K-1}\left(f_{i}\right)\right]
$$

The row complex vector $r^{\mathrm{m}}\left(\mathrm{f}_{\mathrm{i}}\right) \in \mathbb{C}^{1 \times \mathrm{K}}$, and the column complex vector $c^{\mathrm{k}}\left(\mathrm{f}_{\mathrm{i}}\right) \in \mathbb{C}^{\mathrm{M} \times 1}$; thus based on (7), and (8) the spatio-temporal complex matrix $A(f) \in \mathbb{C}^{\mathrm{M} \times \mathrm{K}}$, which represents the collected eigenspectrums from different antennas using different tapers.

The singular value decomposition (SVD) of the matrix $\boldsymbol{A}\left(f_{i}\right)$, produces three different matrices represent the decomposition process as: 


$$
\boldsymbol{A}\left(f_{i}\right)=\boldsymbol{U}\left(f_{i}\right) \boldsymbol{\sigma}\left(f_{i}\right) \boldsymbol{V}\left(f_{i}\right)^{H}
$$

where $\boldsymbol{\sigma}\left(f_{i}\right) \in \mathbb{R}^{M \times K}$, is a diagonal real positive matrix consists of singular values of matrix $\boldsymbol{A}\left(f_{i}\right)$. If $R=\min \{M, K\}$, there will be exactly $R$ nonzero singular values. These singular values are represented by the $\mathrm{R}$ nonzero diagonal elements of the top left $R \times R$ block of $\boldsymbol{\sigma}\left(f_{i}\right)$, where $\sigma_{1}\left(f_{i}\right) \geq$ $\sigma_{2}\left(f_{i}\right) \geq \cdots \geq \sigma_{R}\left(f_{i}\right)>0$. Complex matrix $\boldsymbol{U}(f) \in$ $\mathbb{C}^{M \times M}$ consists of the associated left vectors. Complex matrix $\boldsymbol{V}\left(f_{i}\right) \in \mathbb{C}^{K \times K}$ consists of the associated right vectors; the subscript $H$ denotes the Hermitian transpose. The eigenvalues of the matrix produced from $\boldsymbol{A}\left(f_{i}\right)^{H} \boldsymbol{A}\left(f_{i}\right)$ are $\left|\sigma_{1}\left(f_{i}\right)\right|^{2} \geq\left|\sigma_{2}\left(f_{i}\right)\right|^{2} \geq \cdots \geq\left|\sigma_{R}\left(f_{i}\right)\right|^{2}>0$.

The spectrum estimation using Local-MTM-SVD can be defined based on the singular value squares $\left|\sigma_{1}\left(f_{i}\right)\right|^{2}$, $\left|\sigma_{2}\left(f_{i}\right)\right|^{2}, \ldots,\left|\sigma_{R}\left(f_{i}\right)\right|^{2}$, the number of tapers $K$, and the number of used antennas $M$ as follows:

$$
S_{M T M-S V D}\left(f_{i}\right)=\frac{1}{M K}\left(\sum_{v=1}^{V}\left|\sigma_{v}\left(f_{i}\right)\right|^{2}\right)
$$

where $M K$, is found as the scaling factor of the resulted summation of the different singular value squares, and $V$ represents the number of the singular value squares which should be used in the Local-MTM-SVD spectrum estimation, where $1 \leq V \leq R$. The number of singular values that should be used in the Local-MTM-SVD will be discussed through our result section. The term $S_{M T M-S V D}\left(f_{i}\right)$ represents the spectrum estimation at frequency $f_{i}$ using $K$ tapers, and $M$ antennas, while the term $S_{M T M}^{m}\left(f_{i}\right)$ represents the spectrum estimation that achieved from single antenna using $K$ tapers.

Decision $D E C$ over time interval $L$, and at a specific frequency bin using the MTM when single antenna is used can be formulated as:

$$
D E C_{M T M}^{m}\left(f_{i}\right)=\frac{1}{L} \sum_{l=0}^{L-1} S_{M T M}^{l, m}\left(f_{i}\right)
$$

Thus the decision at a specific frequency bin over the spectrum sensing time duration $L$ when the binary hypothesis $\mathcal{H}_{1}$, is valid, can be rewritten using (2) and (3) in (11) to be as follow:

$$
\begin{aligned}
& \operatorname{DEC} C_{M T M}^{m}\left(f_{i}\right) \\
= & \frac{1}{L} \sum_{l=0}^{L-1} \frac{\sum_{k=0}^{K-1} \lambda_{k}(N, W)\left|\sum_{t=0}^{N-1} v_{t}^{(k)}(N, W)\left(h^{m} s_{t}(l)+w_{t}^{m}(l)\right) e^{-j 2 \pi f_{i} t^{t}}\right|^{2}}{\sum_{k=0}^{K-1} \lambda_{k}(N, W)}
\end{aligned}
$$

When using the periodogram, the decision can be formulated as:

$$
D E C_{P E}^{m}\left(f_{i}\right)=\frac{1}{L} \sum_{l=0}^{L-1} S_{P E}^{l, m}\left(f_{i}\right)
$$

By rewriting (13) using (4), the decision at a specific frequency bin using the periodogram when the binary hypothesis $\mathcal{H}_{1}$, is valid is as follow:

$$
D E C_{P E}^{m}\left(f_{i}\right)=\frac{1}{L N} \sum_{l=0}^{L-1}\left|\sum_{t=0}^{N-1}\left(h^{m} s_{t}(l)+w_{t}^{m}(l)\right) e^{-j 2 \pi f_{i} t}\right|^{2}
$$

Note that (11)-(14), are the decision statistics when single antenna is used.

The decision statistics when number of $M$ antennas are used in the Local-MTM-SVD spectrum sensing at each CR node (sensor) can be redefined using (10) as:

$$
D E C_{M T M-S V D}\left(f_{i}\right)=\frac{1}{L} \sum_{l=0}^{L-1} S_{M T M-S V D}^{l}\left(f_{i}\right)
$$

The detection and false alarm probabilities at each frequency bin using the decision statistics $D E C_{M T M}^{m}\left(f_{i}\right), D E C_{P E}^{m}\left(f_{i}\right)$, and $D E C_{M T M-S V D}\left(f_{i}\right)$ are defined as:

$$
\begin{aligned}
& P_{D}\left(f_{i}\right)=\operatorname{Pr}\left\{\operatorname{DEC}\left(f_{i}\right)>\gamma \mid \mathcal{H}_{1}\right\} \\
& P_{F}\left(f_{i}\right)=\operatorname{Pr}\left\{\operatorname{DEC}\left(f_{i}\right)>\gamma \mid \mathcal{H}_{0}\right\}
\end{aligned}
$$

The threshold $\gamma$, is defined according to the noise variance $\sigma_{\text {noise- } m}^{2}$ when single antenna is used. When a number of antennas are used as in the Local- MTM-SVD, the overall system noise variance $\sigma_{\text {com }}^{2}$ in AWGN can be defined using the noise variances for the different antennas branches $\sigma_{\text {noise }-m}^{2}$ as:

$$
\sigma_{\text {com }}^{2}=\frac{1}{M} \sum_{m=0}^{M-1} \sigma_{\text {noise }-m}^{2}
$$

Thus from (17), the local MTM-SVD as a combining technique averages the noise variance over number of antennas output branches. If the different antennas output have the same receiver noise variance $\sigma_{\text {noise }-m}^{2}$; the resulted combined noise variance will be $\sigma_{\text {noise }-m}^{2}$ as well.

The different techniques decision statistics are calculated at each frequency bin using (1)-(15), and then the probabilities of detection and false alarm can be evaluated by comparing the decision statistic to the predefined threshold over number of realizations using (16).

The overall probability of detection $P_{d}$ is the averaged summation of $P_{D}\left(f_{i}\right)$ over frequency bins from $f_{a}$ to $f_{b}$. The overall probability of false alarm $P_{f}$ is the averaged summation of $P_{F}\left(f_{i}\right)$ over the remained frequency bins. Note that the number of bins contain primary signal is equal to the number of bins which free from primary signal

The joint probability of detection, $Q_{d}$, and the joint probability of false alarm, $Q_{f}$, of the OR rule combining at the CR basestation using $G$ CR nodes (sensors) with identical probabilities of detection and false alarm are given by:

$$
Q_{d}=1-\prod_{g=1}^{G}\left(1-P_{d, g}\right)
$$




$$
Q_{f}=1-\prod_{g=1}^{G}\left(1-P_{f, g}\right)
$$

Where $P_{d, g}, P_{f, g}$ represent the probabilities of detection and false alarm that achievd by the $g^{\text {th }}$ CR node (sensor).

\section{Simulation Results}

The PR user's transmitter uses 64-IFFT with sampling frequency $20 \mathrm{MHz} / T_{S}=0.05 \mu \mathrm{s}$, where $T_{S}$ represents the symbol duration, and transmits QPSK signal from $f_{a}=0.25$ to $f_{b}=0.75$. The $\mathrm{CR}^{\prime}$ node uses 64 -FFT with sampling frequency $20 \mathrm{MHz}$ as well. The performance of the CR user' node is evaluated using $20\left(\mathrm{~N}_{\mathrm{FFT}}\right)=1280$ samples, where spectrum sensing process is carried out every $L=20$ OFDM blocks. Each CR node performs the local MTM-SVD using 1, 2, 3 and 4 antennas. Then 5 CR users relay their decisions as binary digits to the main $\mathrm{CR}$ basestation, which apply OR rule cooperation algorithm to declare the final decision to the $\mathrm{CR}$ network. In all cases of simulations the results are averaged over 10000 simulation runs. The channels considered in the simulation are AWGN with zero mean and variance $\sigma_{\text {noise }-m}^{2}$ using MTM, and periodogram with single antenna, and with $\sigma_{\text {noise }-m}^{2}$ for each antenna using SIMO system, and Rayleigh flat fading. The MTM's parameters chosen her are $N W=4$, and 5 tapers .

Fig. 2 shows the probability of detection versus the probability of false alarm using the local-MTM-SVD technique where $M=1,2,3$ and 4 antennas , $V=1$ in $M=1$ case, and 2 in $M=2,3$, and 4 cases. The channel is assumed as AWGN with $\mathrm{SNR}=-10 \mathrm{~dB}$ per single antenna, and therefore $\mathrm{SNR}=-10 \mathrm{~dB}$ using Local-MTM-SVD as well. The performance is compared with the MTM, and the periodogram using single antenna for both techniques at the same conditions as shown in Table I.

Firstly we note that both local-MTM-SVD with single antenna and MTM spectrum estimation have the same performance at the same system conditions. Secondly the number of singular values squares $(V)$ used in the LocalMTM-SVD should be smaller than or equal to two $(V \leq 2)$ because the improvement in the performance using more than two singular values is not noticeable. Thus, using $\mathrm{V} \leq 2$, achieves good enough performance at the same time minimizes the complexity of the processing by using enough singular values squares.

The percentage of improvement using multi antenna compared to single antenna for the MTM is as follow: $18 \%$ using two antennas with $V=2 ; 23 \%$ using three antennas with $V>1$; and $27 \%$ using four antennas with $V>1$.

The periodogram gives probability of detection as $33 \%$ at the same conditions, thus the local-MTM-SVD outperforms the periodogram by $37 \%$ using single antenna, $55 \%$ using two antennas and $V=2,60 \%$ using three antennas with $V>1$, and finally $64 \%$ using four antennas with $V>1$.

Fig. 3. Shows the probability of detection versus the probability of false alarm for Local-MTM-SVD with $M=1$, $V=1$, and $M=2,3,4$ where $V=2$ and the periodogram when the wireless channel is Rayleigh flat fading with average signal to noise ratio $\mathrm{SNR}=-5 \mathrm{~dB}$. The performance is compared with the MTM, and the periodogram using single antenna for both techniques at the same conditions as shown in Table II.

Fig.4. shows the OR rule joint probability of detection versus the joint probability of false alarm for local-MTM-SVD with 3 antennas and $\mathrm{V}=2$, and for the periodogram when $5 \mathrm{CR}$ users $(G=5)$ at $\mathrm{AWGN}$ with $\mathrm{SNR}=-10 \mathrm{~dB}$ are used. At probability of false alarm $10 \%$, the joint probability of detection is $100 \%$ using the local-MTM-SVD, and $50 \%$ using the periodogram. Thus it is clear that there is a significant improvement in the performance compared to the periodogram.

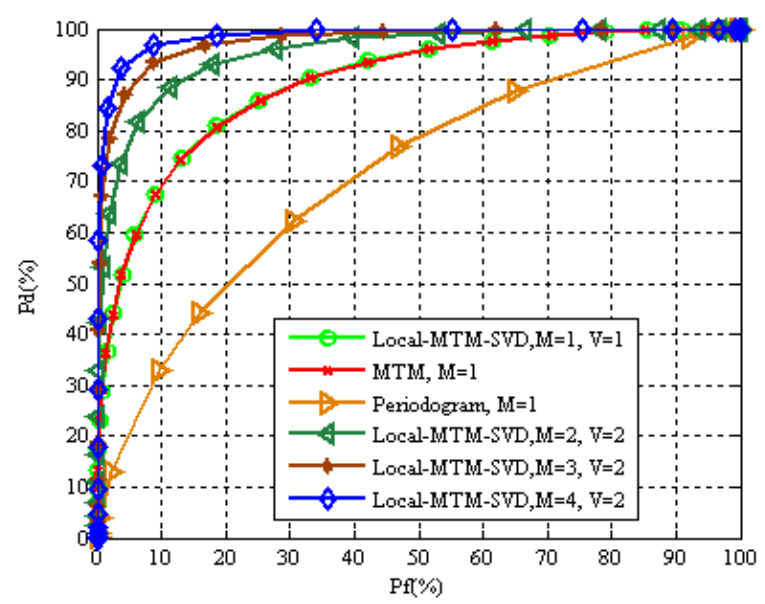

Fig. 2. Probability of detection versus probability of false alarm for LocalMTM-SVD, MTM, and the periodogram at AWGN with $\mathrm{SNR}=-10 \mathrm{~dB}$.

TABLE I. PRobability of Dedection For DifFerent TECHNIQUES AT AWGN (SNR $=-10 \mathrm{~dB}$ ) WhEN FALSE ALARM IS $10 \%$.

\begin{tabular}{|c|c|c|c|c|c|c|}
\hline \multirow{2}{*}{$\begin{array}{c}\text { No. of } \\
\text { antennas }\end{array}$} & \multicolumn{4}{|c|}{ Local-MTM-SVD } & \multirow{2}{*}{ MTM } & \multirow{2}{*}{ Periodogram } \\
\cline { 2 - 7 } & $\boldsymbol{V}=\mathbf{1}$ & $\boldsymbol{V}=\mathbf{2}$ & $\boldsymbol{V}=\mathbf{3}$ & $\boldsymbol{V}=\mathbf{4}$ & & $33 \%$ \\
\hline 1 & $70 \%$ & - & - & - & $70 \%$ & - \\
\hline 2 & $86 \%$ & $88 \%$ & - & - & - & - \\
\hline 3 & $92 \%$ & $93 \%$ & $93 \%$ & - & - & - \\
\hline 4 & $96 \%$ & $97 \%$ & $97 \%$ & $97 \%$ & - & \multicolumn{1}{c}{} \\
\hline
\end{tabular}

TABLE II. PROBABILITY OF DEDECTION FOR DifFERENT TECHNIQUES AT RAYLEIGH FLAT FADING (SNR $=-5 \mathrm{~dB})$ WHEN FALSE ALARM IS 10\%.

\begin{tabular}{|c|c|c|c|c|}
\hline \multirow{2}{*}{$\begin{array}{c}\text { No. of } \\
\text { antennas }\end{array}$} & \multicolumn{2}{|c|}{ Local-MTM-SVD } & \multirow{2}{*}{ MTM } & Periodogram \\
\cline { 2 - 3 } & $\mathbf{V = 1}$ & $\mathbf{V = 2}$ & $75 \%$ & $62 \%$ \\
\hline 1 & $75 \%$ & - & - & - \\
\hline 2 & - & $92 \%$ & - & - \\
\hline 3 & - & $97 \%$ & - & - \\
\hline 4 & - & $99 \%$ & - & \\
\hline
\end{tabular}




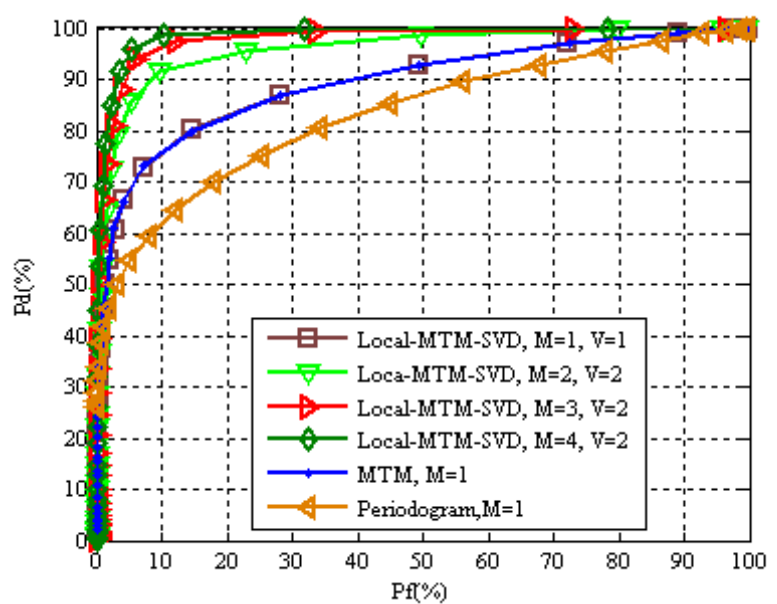

Fig. 3. Probability of detection versus probability of false alarm for LocalMTM-SVD and the periodogram at Rayleigh flat fading with average $\mathrm{SNR}=-5 \mathrm{~dB}$.

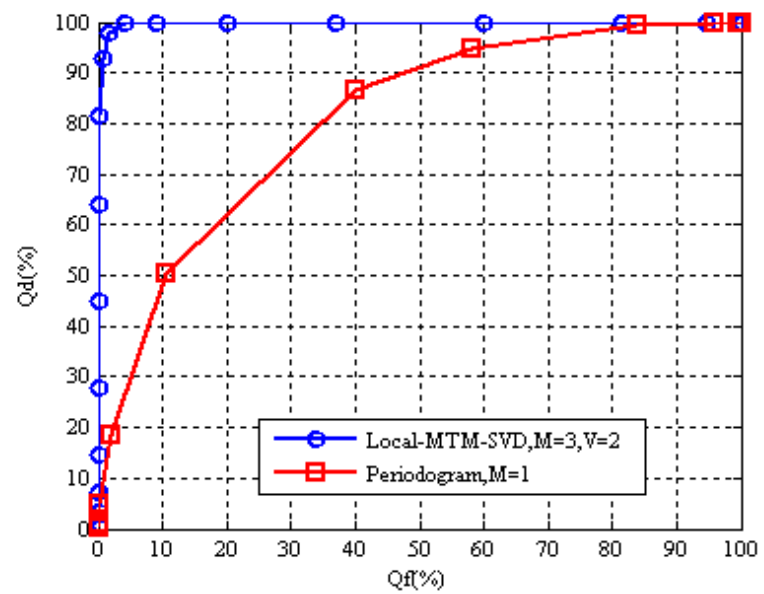

Fig. 4. OR rule Joint Probability of detection versus joint probability of false alarm using Local-MTM-SVD with $M=3$, and $V=2$, and the periodogram using $5 \mathrm{CR}$ users $(G=5)$ at $\mathrm{AWGN}$ with $\mathrm{SNR}=-10 \mathrm{~dB}$.

\section{CONCLUSION}

In this paper, we have proposed the use of local-MTMSVD such that individual node implements the MTM-SVD locally using multi antenna technique for spectrum sensing that uses the opportunistic approach by OFDM-based CR users. The proposed technique improves the overall spectrum sensing process in two ways; firstly it uses MTM which minimizes the spectral leakage and improves the variance of the estimates, and is supported by SVD for denoising; and secondly the binary reporting of the CR nodes(sensors) decisions to the $\mathrm{CR}$ basestation using narrow bandwidth control channel.

The proposed technique has been analyzed and simulated in AWGN, and Rayleigh flat fading at different number of antennas at the $\mathrm{CR}$ nodes and for various number of singular values square. Both $\mathrm{PR}$ user and $\mathrm{CR}$ users were communicating through OFDM-based systems with 64IFFT/FFT.

The number of singular values squares that gives sufficient improvement and minimizes the processing complexity in our case is $V \leq 2$. While the decision threshold is defined by the noise level when $\mathrm{V}$ singular value squares included in decision statistic, we found that removing the lower singular values produces minor changes to the level of the decision threshold but reduces the computation of the required signal processing.

Performing Local-MTM-SVD at each CR node (sensor) significantly increases the probability of detection in AWGN, and Rayleigh flat fading environments. We note when the number of antennas increases, the improvement becomes smaller.

Using the OR cooperation scheme, both periodogram and the proposed technique are affected by the Rayleigh flat fading more than in the AWGN. Furthermore, the proposed technique performance tends to be more robust than the periodogram in the low SNR condition.

Work carried out by our research group is currently focused on the probability of detection and false alarms for MTM in different wireless environments based on statistical analysis.

\section{REFERENCES}

[1] D. J. Thomson, "Spectrum estimation and harmonic analysis," Proc IEEE, vol.70, pp. 1055-1069, September 1982.

[2] M. E. Mann and J .Park, " Global scale modes of surface temperature variability on interannual to century timescales, "Journal of geophysical research, vol.99, pp. 25819-25833, 1994.

[3] M. E. Mann and J .Park, " Oscillatory spatiotemporal signal detection in climate studies: A multiple-taper spectral domain approach," in Advances In Geophysics, R. Dnowska and B. Saltzman, Eds.NewYork: Academic vol.41, pp.1-131, 1999.

[4] S. Haykin, " Cognitive Radio: Brain-Empowered Wireless Communications," IEEE J. Selected Areas in Comm., vol.23, pp.201-220, February 2005 .

[5] J. Ma,G. Ye Lie, and B. Hwang, "Signal Processing in Cognitive Radio," IEEE J. Communications Surveys and tutorials., vol.97,no.5 pp. 805-823, May 2009.

[6] E. Vistotsky, S. Kuffner, and R. Peterson, "On collaborative detection of TV transmissions in support of dynamic spectrum sharing," in Proc. IEEE Symp. New Frontiers in Dynamic Spectrum Access Networks (DySPAN), Baltimore, MD, pp. 338-345, Nov. 2005.

[7] O. A. Alghamdi, and M. A. Abu-Rgheff, " Performance Evaluation of Cognitive Radio Spectrum Sensing Using Multitaper-Singular Value Decomposition," in Proc.IEEE, Crowncom 09, pp. 1-6,Jun. 2009.

[8] O. A. Alghamdi, and M. A. Abu-Rgheff, "Optimal Multitaper Spectrum Sensing in OFDM-Based Cognitive Radio Systems," submitted to IWCMC2010, Caen,France.

[9] S. Haykin and M. Moher, Modern Wireless Communications. USA: Pearson Prentice Hall, 2005.

[10] A. Ghasemi and E. Sousa, "Collaborative spectrum sensing for opportunistic access in fading environments," in Proc. IEEE Symp.New Frontiers in Dynamic Spectrum Access Networks (DySPAN),Baltimore, MD, pp. 131-136, Nov. 2005. 


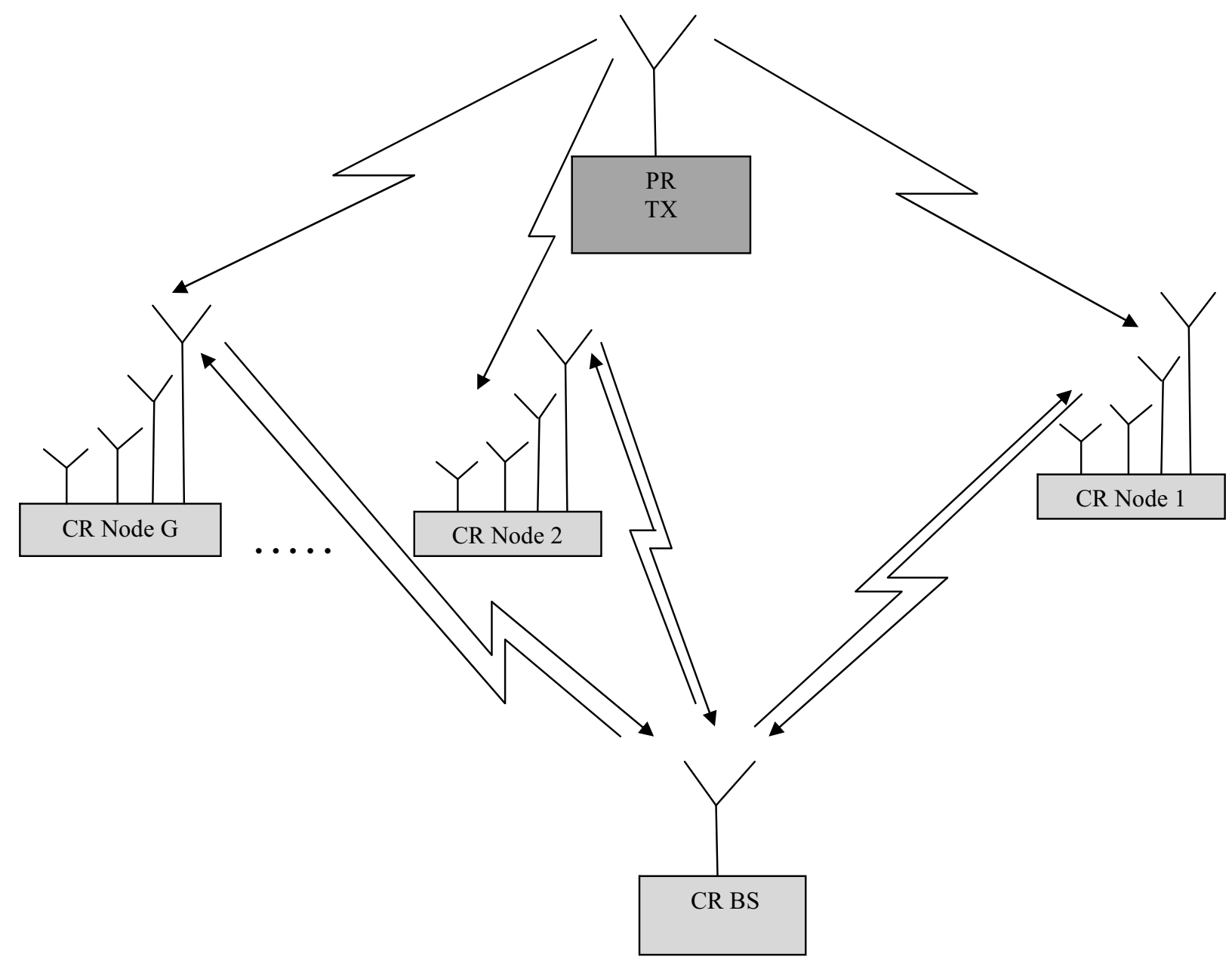

Fig. 1. Number of CR users are sensing the PR signal using Local-MTM-SVD-SIMO technique and retransmit the decision result as a hard decision to the main CR basestation (BS) to perform the cooperation and declare the presence/ or absence of PR signal in the band. 